\title{
Perfil docente y fomento de la cultura del emprendimiento: búsqueda de una relación ${ }^{1}$
}

\section{Vanessa Paola Pertuz-Peralta}

Universidad de Santander, Colombia vanesapertuz@hotmail.com

Astrid Navarro-Rodríguez

Universidad de Santander, Colombia navarro.astrid@gmail.com

\section{Gloria Yasmina Rojas-Caicedo}

Universidad de Santander, Colombia grojas@valledupar.udes.edu.co

Liliana Teresa Quintero

Universidad de Santander, Colombia lquintero@valledupar.udes.edu.co

\section{Resumen}

\section{Palabras clave}

La investigación identifica el perfil del docente basado en competencias que facilita los procesos de aprendizaje del emprendimiento en estudiantes universitarios. La investigación es de tipo descriptivo, con un diseño de campo no experimental y transeccional; se consideró una población de 1195 docentes de las universidades con modalidad presencial de Valledupar (Colombia), de los cuales fueron seleccionados 209, mediante la técnica de muestreo sectorizado y aleatorio simple. Se utilizó un cuestionario de recolección de datos con escala tipo Likert. Según los resultados, los docentes que tienen conocimiento en emprendimiento son facilitadores y comunicadores. Los rasgos de carácter son la capacidad de aprender, la adaptabilidad y la empatía. Son responsables, respetuosos, motivadores e innovadores, pero intolerantes.

Pedagogía universitaria; competencias del docente; formación de docentes; relación profesor-alumno; Valledupar-Colombia (Fuente: Tesauro de la Unesco).

1 Este artículo es producto del proyecto de investigación titulado: “Caracterización del ecosistema emprendedor en las instituciones de educación superior en el municipio de Valledupar", financiado por la convocatoria interna de la Universidad de Santander, año 2013

Recepción: 24-03-2015 | Envío a pares: 13-10-2015 | Aceptación por pares: 17-11-2015 | Aprobación: 07-12-2015 


\title{
The Teacher's Profile and Promoting a Culture of Entrepreneurship: Finding a Connection
}

\begin{abstract}
The study identifies the teacher's profile, based on the skills that facilitate college students learning about entrepreneurship. A descriptive approach was used, with a nonexperimental, cross-sectional design. A population of 1,195 teachers at several on-site universities in Valledupar (Colombia) was considered, and 209 ultimately were chosen through sectored, simple random sampling. A questionnaire with a Likert scale was used to collect the data. The results show that teachers who have knowledge of entrepreneurship are facilitators and communicators. An ability to learn, adaptability and empathy are their character traits. They are responsible, respectful, motivating and innovative, but intolerant.
\end{abstract}

\section{Keywords}

College teaching; teacher's expertise; teacher training; professor-student relationship; Valledupar-Colombia (Source: Unesco Thesaurus). 


\section{Perfil docente e fomento da cultura do empreendimento: busca de uma relação}

Resumo

A pesquisa identifica o perfil do docente baseado em competências que facilitam os processos de aprendizagem do empreendimento em estudantes universitários. A pesquisa é de tipo descritivo, com um desenho de campo não experimental e transeccional; considerou-se uma população de 1.195 docentes das universidades com modalidade presencial de Valledupar (Colômbia), dos quais foram selecionados 209, mediante a técnica de amostragem estratificada e aleatória simples. Utilizou-se um questionamento de coleta de dados com a escala Likert. Segundo os resultados, os docentes que têm conhecimento em empreendimento são facilitadores e comunicadores. Os traços de carácter são a capacidade de aprender, a adaptabilidade e a empatia. São responsáveis, respeitosos, motivadores e inovadores; contudo, são intolerantes.

\section{Palavras-chave}

Pedagogia universitária; competências do docente; formação de docentes; relação professor-aluno; Valledupar; Colômbia (Fonte: Tesauro da Unesco). 
ISSN 0123-1294 | Educ.Educ. Vol. 19. No. 1 | Enero-Abril de 2016 | pp. 29-45.

Universidad de La Sabana | Facultad de Educación

\section{Introducción}

La Ley 1014 de 2006, orientada al fomento de la cultura del emprendimiento en Colombia, plantea que "la formación para el emprendimiento busca el desarrollo de la cultura del emprendimiento con acciones que buscan entre otros la formación en competencias básicas, competencias laborales, competencias ciudadanas y competencias empresariales dentro del sistema educativo formal y no formal y su articulación con el sector productivo" (Congreso de Colombia, 2006). Así, resulta relevante fortalecer las capacidades para la enseñanza del emprendimiento, razón por la cual el presente artículo identifica el perfil del docente formador de emprendedores basado en competencias.

A continuación se describen el marco conceptual de competencias y su relación con el emprendimiento, así como las características de los formadores. Seguidamente, se enuncian los aspectos metodológicos de la investigación, los resultados estadísticos del estudio de campo y las principales conclusiones.

\section{Competencia y emprendimiento}

Una competencia se define como saber hacer en contexto, y contiene tres componentes funda- mentales: el saber (conocimientos), el hacer (habilidades) y el ser (actitudes y valores) (Cultura E Medellín, 2010).

Desde la perspectiva de la investigación, el contexto en el cual se evidencia la formación y el desarrollo de las competencias es el proceso pedagógico realizado por un docente para desarrollar actitudes y habilidades emprendedoras en los estudiantes universitarios; en consecuencia, se adopta el modelo de definición de competencias de "Iceberg" (figura 1) propuesto por Spencer y Spencer (1993), con el objeto de lograr un abordaje práctico desde la formación y evaluación de las competencias del docente formador en emprendimiento. En síntesis, el modelo de Iceberg indica que los conocimientos y las habilidades se encuentran en la parte superior; mientras que en la parte más profunda se localizan el rol social, la imagen de sí mismo, los rasgos y los motivos (Cultura E Medellín, 2010).

\section{Competencias del docente formador en emprendimiento}

El docente formador de emprendedores requiere unas características y unas competencias específicas de manera integral, para el desarrollo efectivo del proceso de enseñanza-aprendizaje, las

Figura 1. Modelo Iceberg de definición de competencias propuesto por Spencer y Spencer (1993)

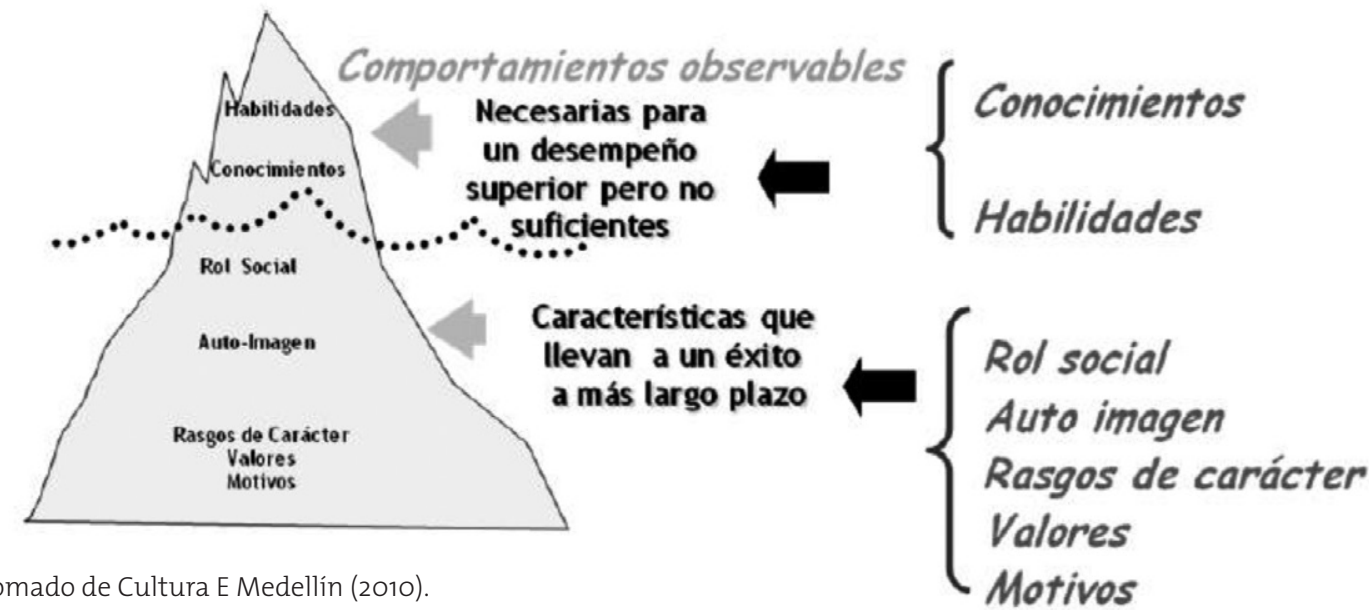

Fuente: tomado de Cultura E Medellín (2010). 
cuales son referenciadas por autores como: Campos Arias y Méndez Parra (2012), Durand (2012), Caldera, Rodríguez y Moreno (2004), Del Solar (2010), Bello (2008), Orti González y Clares López (2007), Cámara de Comercio de Bogotá (2009), Ministerio de Educación de Chile (2009). En el cuadro 1 se describen las características necesarias para el docente formador de emprendedores.

\section{Cuadro 1. Características del docente formador en emprendimiento}

\begin{tabular}{|c|c|c|}
\hline $\mathbf{N}^{\circ}$ & $\begin{array}{l}\text { Caracteristica/ } \\
\text { competencia }\end{array}$ & Definición \\
\hline 1 & Conocimiento & $\begin{array}{c}\text { Genera las condiciones para el desarrollo del aprendizaje significativo en los } \\
\text { estudiantes mediante la transmisión de orientaciones, medios, experiencias, } \\
\text { estrategias y recursos pedagógicos desde un proceso reflexivo de construcción del } \\
\text { conocimiento. }\end{array}$ \\
\hline 2 & $\begin{array}{l}\text { La enseñanza se } \\
\text { centra en el alumno }\end{array}$ & $\begin{array}{l}\text { Orienta al alumno hacia la autonomía y la autodirección del proceso de construcción } \\
\text { de conocimiento, proporcionado las bases requeridas y favoreciendo los espacios de } \\
\text { reflexión, crítica, trabajo en equipo y autoformación. }\end{array}$ \\
\hline 3 & $\begin{array}{l}\text { Promotor del trabajo } \\
\text { en grupo }\end{array}$ & $\begin{array}{l}\text { Asume el grupo como el contexto idóneo para el desarrollo de las habilidades de los } \\
\text { estudiantes, por lo que favorece la solidaridad, la tolerancia, la interacción entre los } \\
\text { estudiantes, la motivación, el sentido de responsabilidad, la discusión y el análisis. }\end{array}$ \\
\hline 4 & Facilitador & $\begin{array}{c}\text { Contribuye a que el estudiante desarrolle sus competencias mediante la motivación, } \\
\text { la orientación y las herramientas de ayuda para el desarrollo efectivo del proceso de } \\
\text { aprendizaje. }\end{array}$ \\
\hline 5 & Problematizador & $\begin{array}{c}\text { Genera preguntas o situaciones que estimulan la capacidad de análisis, la indignación } \\
\text { y la reflexión de los estudiantes como mecanismo para la construcción del } \\
\text { conocimiento. }\end{array}$ \\
\hline 6 & $\begin{array}{c}\text { Identifica } \\
\text { adecuadamente las } \\
\text { necesidades de los } \\
\text { estudiantes }\end{array}$ & $\begin{array}{l}\text { Capacidad de desarrollar el proceso de enseñanza en concordancia con los aspectos } \\
\text { requeridos y las expectativas de los estudiantes. }\end{array}$ \\
\hline 7 & $\begin{array}{l}\text { Trabajan bajo } \\
\text { enfoques sistemáticos } \\
\text { y sustentables }\end{array}$ & $\begin{array}{l}\text { Desarrollan el proceso de enseñanza desde enfoques sistemáticos y sustentables } \\
\text { relacionados con la comunidad local. }\end{array}$ \\
\hline 8 & $\begin{array}{l}\text { Planifican el proceso } \\
\text { de aprendizaje }\end{array}$ & $\begin{array}{l}\text { Establece anticipadamente los objetivos del proceso de enseñanza, definiendo los } \\
\text { contenidos, las metodologías, las estrategias necesarias y los criterios de evaluación } \\
\text { para el dominio de las competencias por parte de los estudiantes. }\end{array}$ \\
\hline 9 & $\begin{array}{l}\text { Evalúan y controlan } \\
\text { los procesos y los } \\
\text { resultados }\end{array}$ & $\begin{array}{c}\text { Incluye dentro de la metodología de proceso de enseñanza-aprendizaje, la realización } \\
\text { de acciones de verificación de los objetivos planteados con el objeto de garantizar la } \\
\text { calidad del proceso y tomar las decisiones de mejora necesarias. }\end{array}$ \\
\hline 10 & $\begin{array}{c}\text { Capacidad de } \\
\text { enseñar en procesos } \\
\text { y contextos prácticos } \\
\text { (reales) / (Ausencia de } \\
\text { clases magistrales) }\end{array}$ & $\begin{array}{l}\text { Fundamenta los procesos de enseñanza-aprendizaje en las experiencias previas } \\
\text { y en la generación de situaciones que permitan al estudiante la construcción del } \\
\text { conocimiento mediante la interacción con el contexto práctico. }\end{array}$ \\
\hline 11 & $\begin{array}{l}\text { Capacidad de trabajar } \\
\text { en equipo }\end{array}$ & $\begin{array}{l}\text { Establece relaciones de cooperación con su entorno con el objeto de lograr metas } \\
\text { comunes de manera efectiva. }\end{array}$ \\
\hline 12 & Comunicación & Capacidad para transmitir efectivamente las ideas a los estudiantes. \\
\hline 13 & Promotor & $\begin{array}{l}\text { Incentiva el desarrollo de trabajos y proyectos donde el estudiante aplica y pone a } \\
\text { prueba sus conocimientos y habilidades. }\end{array}$ \\
\hline
\end{tabular}




\begin{tabular}{|c|c|c|}
\hline $\mathbf{N}^{\circ}$ & $\begin{array}{l}\text { Caracteristica / } \\
\text { competencia }\end{array}$ & Definición \\
\hline 14 & Flexibilidad & $\begin{array}{c}\text { Introduce modificaciones y ajustes a la planificación en función de la evolución del } \\
\text { proceso de enseñanza, integrando los temas y las prácticas de interés por parte de los } \\
\text { estudiantes. }\end{array}$ \\
\hline 15 & Adaptabilidad & $\begin{array}{l}\text { Se muestra receptivo a enfrentar situaciones o realizar actividades diferentes a las } \\
\text { planificadas dentro del proceso de enseñanza. }\end{array}$ \\
\hline 16 & Empatía & $\begin{array}{c}\text { Capacidad de ponerse en el lugar de los estudiantes, comprendiendo sus necesidades y } \\
\text { emociones, y asumiendo una actitud abierta al diálogo y al cambio a fin de lograr una } \\
\text { interacción positiva con los estudiante, }\end{array}$ \\
\hline 17 & $\begin{array}{l}\text { Capacidad de } \\
\text { aprender }\end{array}$ & $\begin{array}{l}\text { Realiza permanente procesos de cualificación y actualización dentro de su disciplina } \\
\text { para obtener un resultado efectivo del proceso enseñanza-aprendizaje. }\end{array}$ \\
\hline 18 & Tolerancia & $\begin{array}{l}\text { Promueve dentro del proceso de enseñanza-aprendizaje el respeto hacia las ideas o } \\
\text { prácticas diferentes o contrarias a las propias. }\end{array}$ \\
\hline 19 & Respeto & $\begin{array}{l}\text { Promueve espacios de discusión y análisis fundamentados en el reconocimiento y la } \\
\text { importancia de las ideas de los involucrados en el proceso de enseñanza-aprendizaje. }\end{array}$ \\
\hline 20 & Responsabilidad & $\begin{array}{l}\text { Promueve dentro del proceso de enseñanza-aprendizaje que los estudiantes asumen } \\
\text { las consecuencias de sus actos. }\end{array}$ \\
\hline 21 & Innovador & $\begin{array}{c}\text { Genera cambios en el desarrollo de los procesos de enseñanza-aprendizaje, } \\
\text { propiciando en los estudiantes una nueva forma de observar el entorno y las } \\
\text { oportunidades que este ofrece. }\end{array}$ \\
\hline 22 & Motivador & Impulsa a los estudiantes para llevar a cabo sus iniciativas. \\
\hline
\end{tabular}

Fuente: elaboración propia (2014).

\section{Metodología}

La investigación sigue una metodología descriptiva, con un diseño no experimental, transeccional, y de campo (Hernández et al., 2010). La población de estudio está constituida por 1.195 docentes de las universidades con modalidad presencial de Valledupar, Cesar (Colombia): Universidad de Santander, Universidad Popular del Cesar y Fundación Universitaria del Área Andina (tabla 1). Mediante la técnica de muestreo sectorizado y aleatorio simple se obtuvo una muestra de 209 docentes (tabla 2).

La técnica de recolección de datos utilizada es la encuesta, se aplica un cuestionario como instrumento de recolección de información, el cual consta de 28 reactivos, de los cuales 11 se adoptaron de DomínguezGuedea, Laros, Domínguez-Guedea (2010), mediante una escala de Likert de cinco opciones de respuesta, siendo o: nunca, 1: casi nunca, 2: a veces, 3: casi siempre, 4: siempre. Se establecieron dos baremos para
Tabla 1. Población de la investigación

\begin{tabular}{|c|c|}
\hline Universidad & $\begin{array}{c}\mathbf{N}^{\circ} \text { de } \\
\text { docentes }\end{array}$ \\
\hline Universidad de Santander & 206 \\
\hline Fundación Universitaria del Área Andina & 140 \\
\hline Universidad Popular del Cesar & 849 \\
\hline Total & 1195 \\
\hline
\end{tabular}

Fuente: elaboración propia (2014).

Tabla 2. Muestra de la investigación

\begin{tabular}{|c|c|}
\hline Universidad & $\begin{array}{c}\mathrm{N}^{\circ} \text { de } \\
\text { docentes }\end{array}$ \\
\hline Universidad de Santander & 66 \\
\hline Fundación Universitaria del Área Andina & 57 \\
\hline Universidad Popular del Cesar & 86 \\
\hline Total & 209 \\
\hline \multicolumn{2}{|c|}{ Error de la muestra $=10 \%$} \\
Confianza $=95 \%$
\end{tabular}

Fuente: elaboración propia (2014). 
el análisis de los datos, uno para interpretación de la media (tabla 3) y otro para la interpretación de la desviación estándar (tabla 4). Los intervalos identificados en las tablas incluyen los límites inferiores cerrados ([Li) y los límites superiores abiertos (LS>) de cada una de las categorías (clases).

Tabla 3. Baremo para la interpretación de la media

\begin{tabular}{|c|c|c|}
\hline \multicolumn{2}{|c|}{ Intervalo } & \multirow{2}{*}{ Categorías (nivel de presencia) } \\
\cline { 1 - 2 }$[\mathrm{li}$ & ls> & Ausente \\
\hline 2,17 & 2,44 & Baja \\
\hline 2,44 & 2,70 & Intermedia \\
\hline 2,70 & 2,96 & Alta \\
\hline 2,96 & 3,23 & Muy alta \\
\hline 3,23 & 3,49 & \\
\hline
\end{tabular}

Fuente: elaboración propia (2014).

Tabla 4. Baremo para la interpretación de la desviación estándar

\begin{tabular}{|c|c|c|}
\hline \multicolumn{2}{|c|}{ Intervalo } & \multirow{2}{*}{ Categorías (nivel de dispersión) } \\
\hline$[\mathrm{li}$ & ls> & Ausente \\
\hline 0,66 & 0,75 & Baja \\
\hline 0,75 & 0,84 & Intermedia \\
\hline 0,84 & 0,93 & Alta \\
\hline 0,93 & 1,02 & Muy alta \\
\hline 1,02 & 1,11 & \\
\hline
\end{tabular}

Fuente: elaboración propia (2014).

\section{Resultados}

En la tabla 5 se enuncian los principales resultados de la investigación.

En la subdimensión conocimiento se evidenciaron los resultados obtenidos en la tabla 5. En el indicador de experticia sobre emprendimiento, el $79 \%$ de los docentes indicó que siempre o casi siempre reconocen quién es y qué hace un emprendedor, y utilizan ejemplos relacionados con el tema de clase; el $2 \%$ manifestó que nunca o casi nunca; mientras el $19 \%$ restante indicó que a veces se identifica con esta característica. En síntesis, se obtuvo una muy alta presencia $(3,23)$ del conocimiento de los docentes, con un nivel intermedio de dispersión de las respuestas obtenidas $(0,84)$.

El resultado obtenido en esta subdimensión concuerda con lo planteado por Durand (2012), quien establece que el docente debe proporcionar a sus alumnos las orientaciones, los medios y recursos pedagógicos necesarios para desencadenar los procesos de aprendizaje mediante la transmisión de saber, conocimiento y experiencia (Cámara de Comercio de Bogotá, 2009), la promoción del aprendizaje significativo (Díaz, Barriga y Hernández, 2002), la creación de las condiciones favorables para que el estudiante despliegue una actividad mental rica y diversa (Del Solar, 2010), la reflexión en el proceso de construcción de conocimiento y un acercamiento concreto al ejercicio de aprender a aprender (Ministerio de Educación de Chile, 2009).

Tabla 5. Resultados de la subdimensión conocimiento

\begin{tabular}{|c|c|c|c|c|c|c|c|c|c|c|c|c|c|c|c|}
\hline \multicolumn{16}{|c|}{ Dimensión: competencias } \\
\hline \multirow{3}{*}{ Subdimensión } & \multirow{3}{*}{ Indicador } & \multicolumn{10}{|c|}{ Escala de Likert por indicador } & \multirow{2}{*}{\multicolumn{2}{|c|}{ Total }} & \multirow{3}{*}{$x$} & \multirow{3}{*}{ s } \\
\hline & & \multicolumn{2}{|c|}{$\mathrm{O}$} & \multicolumn{2}{|c|}{1} & \multicolumn{2}{|c|}{2} & \multicolumn{2}{|c|}{3} & \multicolumn{2}{|c|}{4} & & & & \\
\hline & & $\mathrm{Fa}$ & $\%$ & $\mathrm{Fa}$ & $\%$ & $\mathrm{Fa}$ & $\%$ & $\mathrm{Fa}$ & $\%$ & $\mathrm{Fa}$ & $\%$ & $\mathrm{Fa}$ & $\%$ & & \\
\hline Conocimiento & $\begin{array}{c}\text { Experticia / } \\
\text { conocimiento }\end{array}$ & 1 & $\mathrm{O}$ & 3 & 2 & 39 & 19 & 66 & 32 & 96 & 47 & 205 & 100 & 3,23 & 0,84 \\
\hline
\end{tabular}

Fuente: elaboración propia (2014). 
ISSN 0123-1294 | Educ.Educ. Vol. 19. No. 1 | Enero-Abril de 2016 | pp. 29-45.

Universidad de La Sabana | Facultad de Educación

Tabla 6. Resultados de la subdimensión habilidades

\begin{tabular}{|c|c|c|c|c|c|c|c|c|c|c|c|c|c|c|c|}
\hline \multicolumn{16}{|c|}{ Dimensión: competencias } \\
\hline \multirow{3}{*}{ Subdimensión } & \multirow{3}{*}{ Indicador } & \multicolumn{10}{|c|}{ Escala de Likert por indicador } & \multirow{2}{*}{\multicolumn{2}{|c|}{ Total }} & \multirow{3}{*}{$x$} & \multirow{3}{*}{$\mathrm{s}$} \\
\hline & & \multicolumn{2}{|c|}{ o } & \multicolumn{2}{|c|}{1} & \multicolumn{2}{|c|}{2} & \multicolumn{2}{|c|}{3} & \multicolumn{2}{|c|}{4} & & & & \\
\hline & & $\mathrm{Fa}$ & $\%$ & $\mathrm{Fa}$ & $\%$ & $\mathrm{Fa}$ & $\%$ & $\mathrm{Fa}$ & $\%$ & $\mathrm{Fa}$ & $\%$ & $\mathrm{Fa}$ & $\%$ & & \\
\hline \multirow{11}{*}{ Habilidades } & $\begin{array}{l}\text { La enseñanza } \\
\text { se centra en el } \\
\text { alumno }\end{array}$ & 5 & 2 & 21 & 11 & 61 & 30 & 75 & 36 & 43 & 21 & 205 & 100 & 2,63 & 1,00 \\
\hline & $\begin{array}{l}\text { Promotor del } \\
\text { trabajo en } \\
\text { grupo }\end{array}$ & 1 & 0 & 6 & 3 & 37 & 18 & 74 & 36 & 87 & 43 & 205 & 100 & 3,17 & 0,86 \\
\hline & Facilitador & 1 & 0 & 0 & o & 18 & 9 & 71 & 35 & 115 & 56 & 205 & 100 & 3,46 & 0,70 \\
\hline & Problematizador & 4 & 2 & 5 & 2 & 34 & 17 & 85 & 41 & 77 & 38 & 205 & 100 & 3,11 & 0,88 \\
\hline & $\begin{array}{c}\text { Identifica } \\
\text { adecuadamente } \\
\text { las necesidades } \\
\text { de los } \\
\text { estudiantes }\end{array}$ & 2 & 1 & 14 & 7 & 60 & 29 & 109 & 53 & 20 & 10 & 205 & 100 & 2,64 & 0,79 \\
\hline & $\begin{array}{l}\text { Trabajan bajo } \\
\text { enfoques } \\
\text { sistemáticos y } \\
\text { sustentables }\end{array}$ & 5 & 2 & 14 & 7 & 66 & 32 & 80 & 39 & 40 & 20 & 205 & 100 & 2,66 & 0,95 \\
\hline & $\begin{array}{l}\text { Planifican el } \\
\text { proceso de } \\
\text { aprendizaje }\end{array}$ & 8 & 4 & 20 & 10 & 64 & 31 & 59 & 29 & 54 & 26 & 205 & 100 & 2,64 & 1,09 \\
\hline & $\begin{array}{l}\text { Evalúan y } \\
\text { controlan los } \\
\text { procesos y los } \\
\text { resultados }\end{array}$ & 2 & 1 & 6 & 3 & 33 & 16 & 82 & 40 & 82 & 40 & 205 & 100 & 3,15 & 0,86 \\
\hline & $\begin{array}{c}\text { Capacidad } \\
\text { de enseñar } \\
\text { en procesos } \\
\text { y contextos } \\
\text { prácticos/ } \\
\text { Ausencia de } \\
\text { clase magistral }\end{array}$ & 3 & 1 & 3 & 1 & 36 & 18 & 80 & 39 & 83 & 41 & 205 & 100 & 3,16 & 0,85 \\
\hline & $\begin{array}{l}\text { Capacidad de } \\
\text { trabajar en } \\
\text { equipo }\end{array}$ & 1 & 0 & 2 & 1 & 34 & 17 & 110 & 54 & 58 & 28 & 205 & 100 & 3,08 & 0,73 \\
\hline & Comunicación & 0 & 0 & 2 & 1 & 15 & 7 & 82 & 40 & 106 & 52 & 205 & 100 & 3,42 & 0,67 \\
\hline \multicolumn{2}{|c|}{ Promedio } & 3 & 1 & 8 & 5 & 42 & 20 & 82 & 40 & 70 & 34 & 205 & 100 & 3,01 & 0,85 \\
\hline
\end{tabular}

Fuente: elaboración propia (2014).

Para la subdimensión habilidades se obtuvieron los resultados presentados en la tabla 6. La mayor parte de la población muestra una tendencia positiva frente a las habilidades de un docente que promueve el emprendimiento, lo que se refleja en una alta presencia $(3,01)$, y un nivel intermedio de dispersión (0,85).

En primer lugar, se evidencia que la mayor habilidad del docente es ser facilitador, con una muy alta presencia $(3,46)$ y un nivel de dispersión ausen- te $(0,70)$. El 91\% de los encuestados manifestó que siempre o casi siempre actúa como facilitador en el proceso de enseñanza-aprendizaje, mientras que el 9\% manifestó que a veces, y el o \% que nunca o casi nunca aplican esta habilidad.

El resultado coincide con lo planteado en la revisión de literatura, la cual señala que el docente debe contribuir a que el estudiante desarrolle su vocación emprendedora y sus competencias empresariales mediante la orientación, la recomendación 
y la entrega de herramientas y medios que hagan más fácil su labor (Cámara de Comercio de Bogotá, 2009). Igualmente, el docente debe otorgar una ayuda efectiva en la adquisición de las competencias de los estudiantes (Ministerio de Educación de Chile, 2009), debido a que el emprendedor requiere la motivación y el impulso para llevar a cabo sus iniciativas (Durand, 2012).

En segundo lugar, la comunicación presenta una muy alta presencia $(3,42)$ como habilidad en la población analizada, con un nivel de dispersión ausente en las respuestas $(0,67)$. Frente a este indicador, el 92\% casi siempre o siempre escucha con atención y sabe transmitir sus ideas, mientras el $7 \%$ indicó que a veces, y el 1\% de los encuestados manifestó que nunca o casi nunca desarrolla esta habilidad en su labor docente.

El hallazgo anterior apoya los resultados de la revisión bibliográfica debido a que el docente formador de emprendimiento se caracteriza por la comunicación efectiva (Cámara de Comercio de Bogotá, 2009; Vainrub, 2007), lo que implica la capacidad para gestionar de manera didáctica la información y las destrezas que pretende transmitir a sus estudiantes (Zabalza, 2003).

En tercer lugar, se presenta como habilidad la promoción del trabajo en grupo, con una alta presencia en la población analizada (3,17); no obstante, se evidencia un nivel intermedio de dispersión en las respuestas $(0,86)$. Al respecto, el $79 \%$ casi siempre o siempre posibilita la participación de los estudiantes en las actividades, mientras el 18\% indicó que a veces, y el $3 \%$ de los encuestados manifestó que nunca o casi nunca promueve esta habilidad. Este resultado apoya lo planteado por Del Solar (2010), quien afirma que es indispensable orientar la labor docente hacia el trabajo en equipo, debido a que las habilidades que los estudiantes deben desarrollar para convertirse en emprendedores solo pueden ser aprehendidas en un contexto grupal.
En cuarto lugar, se evidencia una alta presencia de la capacidad de enseñar en procesos y contextos prácticos (reales) (3,16), caracterizada por la ausencia de clases magistrales, con un nivel intermedio de dispersión frente a esta habilidad (0,85). El 80\% de los docentes casi siempre o siempre generan espacios de práctica donde los estudiantes aprenden en contextos reales, mientras el $18 \%$ indicó que a veces, y el $2 \%$ de los encuestados manifestó que nunca o casi nunca fomenta esta habilidad en su labor docente.

Frente al resultado anterior, la literatura plantea que para los emprendedores son importantes las actividades prácticas, lo que no significa la ausencia de actividades de reflexión y análisis, de argumentación, de investigación (Durand, 2012). La formación del docente debe ir ligada a un mayor acceso a contextos reales, para poner en práctica el conocimiento; la contextualización constituye una condición necesaria para la formación de competencias (Jaramillo, 2008). Así, el docente debe canalizar los procesos de aprendizaje partiendo de las experiencias previas de la vida de los alumnos, las experiencias de su entorno familiar y sus inquietudes hacia la vida laboral (Del Solar, 2010).

En quinto lugar, se encontró que los docentes presentan un alto nivel de desarrollo de habilidad para evaluar y controlar los procesos y los resultados $(3,15)$, con un nivel intermedio de dispersión en las respuestas (o,86). Al respecto, el $80 \%$ casi siempre o siempre orienta la labor docente a obtener objetivos y metas, mientras el $16 \%$ indicó que a veces, y el $4 \%$ de los encuestados manifestó que nunca o casi nunca se identifica con esta habilidad.

Respecto a esta habilidad, la literatura plantea que, además de la evaluación al finalizar el proceso, es necesario evaluar durante el mismo para tener información respecto a los avances y efectuar los ajustes y las modificaciones necesarias (Durand, 2012); lo anterior garantiza la calidad del proceso de enseñanza-aprendizaje (Zabalza, 2003). 
En sexto lugar, se evidencia una alta presencia de la habilidad de problematizador $(3,11)$ en los docentes, con un nivel intermedio de dispersión $(0,88)$. $\mathrm{El} 79 \%$ casi siempre o siempre promueve en el estudiante la búsqueda de diversas alternativas para la solución de un problema dado, mientras el $17 \%$ indicó que a veces, y el $4 \%$ de los encuestados manifestó que nunca o casi nunca fomenta esta habilidad en su labor docente.

Respecto a este indicador, la literatura plantea que la función esencial del docente consiste en generar preguntas que activen el proceso de indagación y análisis en los estudiantes, "sin las preguntas generadas por el docente, el proceso de enseñanza sería unidireccional y el papel de los estudiantes sería irrelevante" (Hernández y Lacuesta, s.f.). Adicionalmente, según Díaz, Barriga y Hernández (2002), el docente debe pensar críticamente su práctica, tomando decisiones y solucionando problemas en su clase.

En séptimo lugar, se evidencia en los docentes la capacidad de trabajar en equipo, con una alta presencia $(3,08)$ y un nivel ausente de dispersión en las respuestas $(0,73)$. El $82 \%$ de los docentes manifestó que siempre o casi siempre establece relaciones de cooperación y preocupación al trabajar con otros para conseguir metas comunes, frente a un $17 \%$ que a veces promueve esta habilidad y un $1 \%$ que nunca o casi nunca la promueven.

El resultado anterior coincide con lo planteado por Durand (2012), quien afirma que "el trabajo presencial y en equipo es indispensable en la formación de emprendedores". Consecuentemente, en la formación emprendedora es fundamental el trabajo en equipo, y la búsqueda y obtención de apoyo (Filion, 2000).

En octavo lugar, se evidencian deficiencias en la habilidad relacionada con el trabajo bajo enfoques sistemáticos y sustentables, con una baja presencia en la población analizada $(2,66)$; no obstante, en este indicador se evidencia una alta dispersión en las respuestas (0,95). El 59\% manifestó que casi siempre o siempre trabajan con enfoques sistemáticos y sustentables relacionados con la comunidad, mientras el 32\% indicó que a veces, y el $9 \%$ de los encuestados nunca o casi nunca desarrolla esta habilidad en su labor docente.

El resultado anterior difiere de lo planteado en la literatura considerando que los docentes que forman emprendedores se caracterizan porque "trabajan con enfoques sistemáticos y sustentables relacionados con la comunidad local" (Durand, 2012).

En noveno lugar, se evidencia en los docentes un bajo nivel para planificar el proceso de aprendizaje $(2,64)$, con un muy alto nivel de dispersión en las respuestas $(1,09)$. Solo el $55 \%$ de los encuestados manifestó que siempre o casi siempre planifican la actividad educativa de forma coordinada con el resto del profesorado, mientras que el 31\% manifestó que a veces, y el 14\% que nunca o casi nunca desarrolla esta habilidad.

El resultado anterior difiere de lo planteado en la revisión de la literatura, debido a que el docente debe planificar el proceso de enseñanza-aprendizaje, lo que implica conocer las competencias de entrada y de salida de sus estudiantes (Tobón, 2006), tomando decisiones relativas a las estrategias, las actividades y su secuencia didáctica en torno a las metodologías de enseñanza-aprendizaje (Zabalza, 2003); seleccionando los escenarios, las herramientas y los métodos de trabajo (Cámara de Comercio de Bogotá, 2009); definiendo cada una de las etapas del desarrollo de la experiencia, en la que existen estrategias generales, criterios de evaluación y roles de los estudiantes (Hernández y Lacuesta, s. f.).

En décimo lugar, en los docentes se encontró una baja presencia de la habilidad para identificar adecuadamente las necesidades de los estudiantes $(2,64)$, con un bajo nivel de dispersión en las respuestas (o,79). Al respecto, el $63 \%$ de los docentes manifestó que siempre o casi siempre identifican 
las expectativas de los estudiantes, tanto desde de la perspectiva académica como desde la del emprendimiento; mientras $29 \%$ indicó que a veces, y el $8 \%$ que nunca o casi nunca desarrollan esta habilidad.

Los resultados discrepan de lo encontrado en la literatura; según la cual, el docente formador de emprendedores tiene la capacidad de detectar oportunidades (Alcaraz, 2006; Filion, 2000), lo que implica identificar las expectativas del estudiante (Cámara de Comercio de Bogotá, 2009). Así, el docente debe tener la habilidad de conocer quiénes son los estudiantes y comprender cómo aprenden para diseñar una formación de acuerdo con sus necesidades (Durand, 2012).

En último lugar, la habilidad con más baja presencia en los docentes se asocia con la enseñanza centrada en el alumno (2,63); no obstante, se observa un alto nivel de dispersión en las respuestas obtenidas $(1,00)$. Frente a este indicador, solo el $57 \%$ de los docentes siempre o casi siempre realizan actividades o casos seleccionados por los estudiantes, o promueven procesos de autoevaluación y coevaluación, mientras el $30 \%$ a veces y $13 \%$ nunca o casi nunca desarrolla esta habilidad.

Frente al hallazgo anterior, la teoría plantea que "el docente debe distanciarse consciente y progresivamente de constituirse en el centro de la clase" (Del Solar, 2010), mediante la entrega de las indicaciones precisas para que los estudiantes desarrollen su trabajo con autonomía (Díaz, Barriga y Hernández, 2002). En el mismo sentido, Durand (2012) y Jaramillo (2008) plantean que el docente debe asumir el rol de conductor o moderador de procesos (Cámara de Comercio de Bogotá, 2009), lo cual demanda un rol diferente al tradicional, donde el centro del proceso sea el estudiante (Díaz, Barriga y Hernández, 2002).

En la subdimensión rol social se evidenciaron los resultados presentados en la tabla 7. En el indicador asociado al rol de promotor, el $65 \%$ de los docentes indicaron que siempre o casi siempre incentivan el desarrollo de trabajos y proyectos donde el estudiante aplica y pone a prueba su intención emprendedora, el $28 \%$ restante indicó que a veces asume el rol de promotor en su ejercicio docente, mientras el $7 \%$ manifestó que nunca o casi nunca. En sintesis, se obtuvo una intermedia presencia $(2,82)$ del rol de promotor en los docentes analizados, con un nivel intermedio de dispersión de las respuestas obtenidas $(0,91)$.

Respecto a esta subdimensión, la teoría plantea que el docente formador de emprendedores se caracteriza por incentivar el desarrollo de trabajos y proyectos donde el estudiante aplica y pone a prueba su iniciativa empresarial (Cámara de Comercio de Bogotá, 2009).

Tabla 7. Resultados de la subdimensión rol social

\begin{tabular}{|c|c|c|c|c|c|c|c|c|c|c|c|c|c|c|c|}
\hline \multicolumn{16}{|c|}{ Dimensión: competencias } \\
\hline \multirow{3}{*}{ Subdimensión } & \multirow{3}{*}{ Indicador } & \multicolumn{10}{|c|}{ Escala de Likert por indicador } & \multirow{2}{*}{\multicolumn{2}{|c|}{ Total }} & \multirow{3}{*}{$x$} & \multirow{3}{*}{ S } \\
\hline & & \multicolumn{2}{|c|}{ o } & \multicolumn{2}{|c|}{1} & \multicolumn{2}{|c|}{2} & \multicolumn{2}{|c|}{3} & \multicolumn{2}{|c|}{4} & & & & \\
\hline & & $\mathrm{Fa}$ & $\%$ & $\mathrm{Fa}$ & $\%$ & $\mathrm{Fa}$ & $\%$ & $\mathrm{Fa}$ & $\%$ & $\mathrm{Fa}$ & $\%$ & $\mathrm{Fa}$ & $\%$ & & \\
\hline Rol social & Promotor & 1 & $\mathrm{O}$ & 14 & 7 & 57 & 28 & 81 & 40 & 52 & 25 & 205 & 100 & 2,82 & 0,91 \\
\hline
\end{tabular}

Fuente: elaboración propia (2014). 
Tabla 8. Resultados de la subdimensión rasgos de carácter

\begin{tabular}{|c|c|c|c|c|c|c|c|c|c|c|c|c|c|c|c|}
\hline \multicolumn{16}{|c|}{ Dimensión: competencias } \\
\hline \multirow{3}{*}{ Subdimensión } & \multirow{3}{*}{ Indicador } & \multicolumn{10}{|c|}{ Escala de Likert por indicador } & \multirow{2}{*}{\multicolumn{2}{|c|}{ Total }} & \multirow{3}{*}{$x$} & \multirow{3}{*}{ S } \\
\hline & & \multicolumn{2}{|c|}{ O } & \multicolumn{2}{|c|}{1} & \multicolumn{2}{|c|}{2} & \multicolumn{2}{|c|}{3} & \multicolumn{2}{|c|}{4} & & & & \\
\hline & & $\mathrm{Fa}$ & $\%$ & $\mathrm{Fa}$ & $\%$ & $\mathrm{Fa}$ & $\%$ & $\mathrm{Fa}$ & $\%$ & $\mathrm{Fa}$ & $\%$ & $\mathrm{Fa}$ & $\%$ & & \\
\hline \multirow{4}{*}{$\begin{array}{l}\text { Rasgos del } \\
\text { carácter }\end{array}$} & Flexibilidad & 1 & o & 4 & 2 & 36 & 18 & 95 & 46 & 69 & 34 & 205 & 100 & 3,11 & 0,78 \\
\hline & Adaptabilidad & $\mathrm{O}$ & O & 1 & $\mathrm{O}$ & 24 & 12 & 73 & 36 & 107 & 52 & 205 & 100 & 3,40 & 0,71 \\
\hline & Empatía & $\mathrm{O}$ & $\mathrm{O}$ & 3 & 1 & 19 & 9 & 89 & 44 & 94 & 46 & 205 & 100 & 3,34 & 0,71 \\
\hline & $\begin{array}{c}\text { Capacidad de } \\
\text { aprender }\end{array}$ & 1 & $\mathrm{O}$ & 2 & 1 & 17 & 8 & 60 & 30 & 125 & 61 & 205 & 100 & 3,49 & 0,73 \\
\hline \multicolumn{2}{|c|}{ Promedio } & 1 & $\mathrm{O}$ & 3 & 1 & 24 & 12 & 79 & 39 & 98 & 48 & 205 & 100 & 3,33 & 0,73 \\
\hline
\end{tabular}

Fuente: elaboración propia (2014).

Para la subdimensión rasgos del carácter, se obtuvieron los resultados presentados en la tabla 8. La mayor parte de la población muestra una tendencia positiva frente a los rasgos del carácter de un docente que promueve el emprendimiento, hecho que se refleja en una muy alta presencia $(3,33)$ de la subdimensión analizada, con un nivel ausente de dispersión en las respuestas obtenidas (0,73).

En primer lugar, se evidencia que en los docentes analizados el mayor rasgo del carácter es la capacidad de aprender, con una muy alta presencia $(3,49)$ y un ausente nivel de dispersión $(0,73)$. Al respecto, $91 \%$ manifestó que siempre o casi siempre indagan, buscan información y tienen inquietud permanente por conocer más, mientras que el $8 \%$ a veces, y el 1\% nunca o casi nunca desarrollan este rasgo.

En acuerdo con el resultado anterior, la revisión de literatura plantea que "la primera y más importante premisa que debe tener en cuenta quien desde la docencia busca promover y formar en emprendimiento, es que la capacidad de aprender es ilimitada. Esto es fundamental, tanto desde la perspectiva del docente, como del estudiante" (Cámara de Comercio de Bogotá, 2009).

En segundo lugar, la adaptabilidad es el rasgo de carácter presente en los docentes, con una muy alta presencia $(3,40)$ y un nivel de dispersión ausen- te $(0,71)$. El $88 \%$ manifestó que siempre o casi siempre se muestra abierto a enfrentar situaciones distintas a las que está acostumbrado, mientras el $12 \%$ indicó que a veces, y o\% que nunca o casi nunca se identifica con este rasgo.

En consonancia con el resultado anterior, Vainrub (2007) plantea que el docente formador de emprendedores se adapta a los cambios, es decir, se muestra receptivo a enfrentar situaciones o realizar actividades diferentes a las planificadas dentro del proceso de enseñanza.

En tercer lugar, la empatía se identifica como un rasgo del carácter de los docentes, evidenciando una muy alta presencia $(3,34)$ y un nivel de dispersión ausente en las respuestas (0,71). Frente a este indicador, el 90\% de los docentes tienen la capacidad de ponerse en el lugar de sus alumnos y comprender sus motivos, el $9 \%$ indicó que a veces, y el $1 \%$ que nunca o casi nunca se identifica con este rasgo del carácter.

En acuerdo con el resultado anterior, la teoría propone que el docente formador de emprendedores debe tener la capacidad de relacionarse asertivamente con los demás, poniéndose en el lugar del otro, con una actitud abierta al diálogo y al cambio (Durand, 2012); debe comprender las emociones, necesidades e intereses de otros y procurar su apro- 
piada conciliación (Bedoya y Varela, 2007). Así, la misión del profesor del futuro deberá estar orientada a diseñar adecuados sistemas de gestión de conocimiento, con un adecuado respaldo emocional que permita al alumno optimizar al máximo los recursos empleados en su periodo de formación (Orti y Clares, 2007).

En último lugar, se encontró una alta presencia del rasgo del carácter asociado a la flexibilidad $(3,11)$, con un bajo nivel de dispersión en la población analizada (o,78). El 80\% manifestó que siempre o casi siempre brindan espacios en la clase que permiten integrar temas y prácticas de interés por parte de los estudiantes, o confronta sus opiniones con las de los alumnos buscando establecer un acuerdo intermedio, mientras $18 \%$ manifestó que a veces y el $2 \%$ que nunca o casi nunca desarrollan este rasgo del carácter.

El resultado anterior apoya lo planteado por Del Solar (2010), quien afirma que el docente formador de emprendedores sabe introducir modificaciones a la programación según la evolución de cada actividad, mediante una estructura flexible, pero claramente delimitada. Adicionalmente, Durand (2012) plantea que es fundamental que los profesores consideren a los estudiantes como sujetos capaces de construir sus aprendizajes a partir de tareas significativas y prácticas fuera del aula, con el objeto de establecer de manera participativa las estrategias pedagógicas (Jaramillo, 2008).
Para la subdimensión valores, se obtuvieron los resultados presentados en la tabla 9; estos indican que la mayor parte de la población estudiada muestra una tendencia intermedia frente a los valores de un docente que promueve el emprendimiento, hecho que se refleja en una intermedia presencia $(2,80)$ de la subdimensión analizada, con un nivel intermedio de dispersión en las respuestas $(0,90)$.

En primer lugar, se evidencia que en los docentes analizados el valor predominante es la responsabilidad, con una muy alta presencia $(3,41)$ y un ausente nivel de dispersión (0,70). El 91\% de los encuestados manifestó que siempre o casi siempre promueve que los estudiantes asuman las consecuencias de sus actos, mientras que el $8 \%$ manifestó que a veces, y el $1 \%$ que nunca o casi nunca se identifica con este valor.

El resultado anterior coincide con lo planteado por Durand (2012), quien afirma que los docentes formadores de emprendedores asumen y transmiten la perspectiva de que el éxito se logra con base en el trabajo personal, enfrentando con empeño, constancia y ética los obstáculos que se presentan en el medio.

En segundo lugar, se encontró una presencia intermedia del respeto como valor en los docentes analizados (2,83), con un nivel intermedio de dispersión en las respuestas $(0,90)$. Frente a este indicador,

Tabla 9. Resultados de la subdimensión valores

\begin{tabular}{|c|c|c|c|c|c|c|c|c|c|c|c|c|c|c|c|}
\hline \multicolumn{16}{|c|}{ Dimensión: competencias } \\
\hline \multirow{3}{*}{ Subdimensión } & \multirow{3}{*}{ Indicador } & \multicolumn{10}{|c|}{ Escala de Likert por indicador } & \multicolumn{2}{|c|}{ Total } & \multirow[t]{2}{*}{$x$} & \multirow[t]{2}{*}{$\mathrm{s}$} \\
\hline & & $\mathrm{O}$ & 1 & 2 & 3 & 4 & & & & & & & & & \\
\hline & & $\mathrm{Fa}$ & $\%$ & $\mathrm{Fa}$ & $\%$ & $\mathrm{Fa}$ & $\%$ & $\mathrm{Fa}$ & $\%$ & $\mathrm{Fa}$ & $\%$ & $\mathrm{Fa}$ & $\%$ & & \\
\hline \multirow{3}{*}{ Valores } & Tolerancia & 18 & 9 & 36 & 18 & 67 & 33 & 62 & 30 & 22 & 10 & 205 & 100 & 2,17 & 1,11 \\
\hline & Respeto & 4 & 2 & 13 & 6 & 39 & 19 & 106 & 52 & 43 & 21 & 205 & 100 & 2,83 & 0,90 \\
\hline & Responsabilidad & $\mathrm{O}$ & $\mathrm{O}$ & 3 & 1 & 16 & 8 & 80 & 39 & 106 & 52 & 205 & 100 & 3,41 & 0,70 \\
\hline \multicolumn{2}{|c|}{ Promedio } & 7 & 4 & 17 & 8 & 41 & 20 & 83 & 40 & 57 & 28 & 205 & 100 & 2,80 & 0,90 \\
\hline
\end{tabular}

Fuente: elaboración propia (2014) 
el $73 \%$ señaló que casi siempre o siempre promueven la discusión entre sus estudiantes, mientras el 19\% indicó que a veces, y el $8 \%$ de los encuestados manifestó que nunca o casi nunca se identifica con el valor del respeto en el desarrollo de su labor docente.

En contraste con el resultado anterior, la literatura propone que los docentes son fuentes de apoyo, de aliento y orientación a sus estudiantes para la consecución de las metas que se proponen (Bello, 2008). Así, el docente formador de emprendimiento sobresale por su capacidad de inspirar confianza y credibilidad (Cámara de Comercio de Bogotá, 2009); es un docente reconocido, apreciado y exitoso (Cámara de Comercio de Bogotá, 2009); y respetuoso (Orti y Clares, 2007).

En último lugar, se evidencia un nivel ausente de tolerancia en los docentes (2,17); no obstante, se percibe un muy alto nivel de dispersión frente a este valor en la población analizada (1,11). El $40 \%$ de los docentes casi siempre o siempre permite que sus estudiantes corrijan o revisen el trabajo de otro compañero, mientras el $33 \%$ indicó que a veces, y el $27 \%$ de los encuestados manifestó que nunca o casi nunca promueve este valor en el desarrollo de su labor docente.

El resultado anterior difiere de lo planteado en la literatura, según la cual el docente debe establecer un clima de relación basado en la confianza, la seguridad y la aceptación mutuas, favoreciendo la curiosidad, la capacidad de sorprenderse y el gusto por aprender (Del Solar, 2010). Asimismo, el docente formador de emprendedores se caracteriza por tener la capacidad de ponerse en el lugar de los alumnos y comprender sus motivos (Orti y López, 2007).

Para la subdimensión motivos, se obtuvieron los resultados presentados en la tabla 10. Los resultados indican que la mayor parte de la población muestra una tendencia positiva frente a los motivos de un docente que promueve el emprendimiento, hecho que se refleja en una muy alta presencia $(3,34)$ de la subdimensión analizada, con un nivel ausente de dispersión en las respuestas obtenidas (o,69).

En primer lugar, se evidencia en los docentes la capacidad de ser motivador, con una muy alta presencia $(3,34)$ y un nivel de dispersión ausente $(0,72)$. El $87 \%$ de los encuestados manifestó que siempre o casi siempre impulsa a sus estudiantes para llevar a cabo sus iniciativas, mientras que el 12\% manifestó que a veces, y el 1\% que nunca o casi nunca aplican esta capacidad.

El resultado anterior coincide con lo planteado en la literatura al afirmar que el docente formador de emprendedores debe orientar a los estudiantes para que se automotiven y tomen conciencia de su plan de vida y autorrealización (Jaramillo, 2008), mediante la construcción de un ambiente que propicie interés y confianza en el estudiante alrededor del emprendimiento como opción de vida (Cámara de Comercio de Bogotá, 2009).

Tabla 10. Resultados de la subdimensión motivos

\begin{tabular}{|c|c|c|c|c|c|c|c|c|c|c|c|c|c|c|c|}
\hline \multicolumn{16}{|c|}{ Dimensión: competencias } \\
\hline \multirow{3}{*}{ Subimensión } & \multirow{3}{*}{ Indicador } & \multicolumn{10}{|c|}{ Escala de Likert por indicador } & \multirow{2}{*}{\multicolumn{2}{|c|}{ Total }} & \multirow{3}{*}{$x$} & \multirow{3}{*}{$\mathrm{s}$} \\
\hline & & \multicolumn{2}{|c|}{$\mathrm{O}$} & \multicolumn{2}{|c|}{1} & \multicolumn{2}{|c|}{2} & \multicolumn{2}{|c|}{3} & \multicolumn{2}{|c|}{4} & & & & \\
\hline & & $\mathrm{Fa}$ & $\%$ & $\mathrm{Fa}$ & $\%$ & $\mathrm{Fa}$ & $\%$ & $\mathrm{Fa}$ & $\%$ & $\mathrm{Fa}$ & $\%$ & $\mathrm{Fa}$ & $\%$ & & \\
\hline Motivos & Innovador & $\mathrm{O}$ & 0 & 1 & $\mathrm{O}$ & 21 & 10 & 93 & 46 & 90 & 44 & 205 & 100 & 3,33 & 0,67 \\
\hline & Motivador & $\mathrm{O}$ & $\mathrm{O}$ & 2 & 1 & 24 & 12 & 81 & 40 & 98 & 47 & 205 & 100 & 3,34 & 0,72 \\
\hline \multicolumn{2}{|c|}{ Promedio } & $\mathrm{O}$ & 0 & 2 & 1 & 22 & 11 & 87 & 43 & 94 & 45 & 205 & 100 & 3,34 & 0,69 \\
\hline
\end{tabular}

Fuente: elaboración propia (2014) 
En segundo lugar, se evidencia una muy alta presencia del motivo relacionado con ser innovador (3,33), con un nivel de dispersión ausente en las respuestas $(0,67)$. El $90 \%$ de los docentes casi siempre o siempre propone y encuentra formas nuevas y eficaces de facilitar el aprendizaje, mientras el $10 \%$ indicó que a veces, y el o\% de los encuestados manifestó que nunca o casi nunca actúa bajo este motivo.

El hallazgo anterior apoya los resultados de la revisión bibliográfica, según la cual, el docente formador de emprendedores debe tener iniciativa, capacidad de innovar (Cámara de Comercio de Bogotá, 2009), no temer a los cambios (Durand, 2012) y salir de lo convencional, agregando valor al proceso (Alcaraz, 2006).

\section{Conclusiones}

La investigación realizada permite concluir los siguientes aspectos: primero, se identificó que los docentes tienen un muy alto nivel de conocimiento frente al emprendimiento.

Segundo, las habilidades características de los docentes se asocian con ser facilitadores y comunicativos, con muy alta presencia. Adicionalmente, presentan un alto nivel de habilidad para la promoción del trabajo en grupo, la enseñanza en contextos prácticos, la evaluación, el control de los procesos y los resultados, así como la capacidad de problematizar y trabajar en equipo.
En contraste, se observa un bajo nivel de las habilidades correspondientes al trabajo bajo enfoques sistemáticos y sustentables relacionados con la comunidad local, así como para planificar el proceso de aprendizaje, identificar adecuadamente las necesidades de sus estudiantes, y, en consecuencia, centrar la enseñanza en el estudiante.

Tercero, se evidencia en los docentes analizados un nivel intermedio de capacidad para ejercer el rol social de promotor dentro del proceso de enseñanza-aprendizaje.

Cuarto, los rasgos de carácter de los docentes analizados se asocian con una muy alta presencia de la capacidad de aprender, la adaptabilidad y la empatía. Así como con la flexibilidad, aspecto en el cual se obtiene una alta presencia en los docentes analizados.

Quinto, los valores de los docentes analizados corresponden a la responsabilidad, aspecto en el cual se evidencia una muy alta presencia; adicionalmente, los docentes se identifican, en un nivel intermedio, con el valor del respeto. No obstante, se encontró que estos son intolerantes en el ejercicio de la enseñanza-aprendizaje, hecho que se evidencia en la ausencia del indicador.

Sexto, los docentes se identifican con la capacidad de ser motivadores e innovadores, como motivos dentro del ejercicio de enseñanza-aprendizaje, aspectos en los cuales se obtuvo una muy alta presencia.

\section{Referencias}

Alcaraz, R. (2006). El emprendedor de éxito. México: McGraw Hill.

Bedoya, O. L. y Varela, R. (2007). Modelo conceptual de desarrollo empresarial basado en competencias. Cali: Universidad ICESI.

Bello, M. E. (2008). Competencias del docente para la formación de capacidad emprendedora en la Universidad Metropolitana. Caracas: Departamento de Didáctica, Universidad Metropolitana. 
ISSN $0123-1294$ | Educ.Educ. Vol. 19. No. 1 | Enero-Abril de 2016 | pp. 29-45.

Universidad de La Sabana | Facultad de Educación

Caldera, J., Rodríguez, F. y Moreno, J. (2004). Manual guía para agentes multiplicadores en estímulo y desarrollo de capacidades emprendedoras. Medellín: CAF, Universidad Metropolitana, Infoem, Servicio de Atención al Emprendedor.

Cámara de Comercio de Bogotá (2009). El docente como promotor y formador del emprendimiento. Guía básica. Programa de emprendimiento en la región Bogotá, Cundinamarca. Bogota D.C.: Cámara de Comercio de Bogotá.

Campos Arias, O. R. y Méndez Parra, G. C. (2012). La enseñanza del emprendimiento a partir del aprendizaje basado en problemas (ABP) en la educación media técnica. Tesis para optar al título de Magíster en Ciencias de la Educación, Facultad de Educación, Maestría en Ciencias de la Educación, Universidad de la Amazonia, Florencia.

Congreso de Colombia (2006). Ley 1014 de 2006. De fomento a la cultura del emprendimiento. Bogota D.C.: Congreso de Colombia.

Cultura E Medellín. (2010). Programa de fortalecimiento al sistema de emprendimiento de Medellín - Guía del docente. Medellín: Cuidad E, líder en empredimiento, Comfama, Alcaldía de Medellín.

Del Solar, S. (2010). Emprendedores en el aula. Guía para la formación en valores y habilidades sociales de docentes y jóvenes emprendedores. Santiago: Fondo Multilateral de Inversiones del Banco Interamericano de Desarrollo.

Díaz, F., Barriga, A. y Hernández, G. (2002). Estrategias docentes para un aprendizaje significativo. Una interpretación constructivista. México: McGraw Hill.

Domínguez-Guedea, M., Laros, J. y Domínguez-Guedea, R. (2010). Validación de medidas para evaluar habilidades de conducción y evaluación en profesores universitarios. Relieve, 16 (1) 1-12.

Durand, M. C. (2012). Ava Alencastre. Perú: Centro de Competitividad y Estudios Estratégicos de IPAE Acción Empresarial.

Durand, M. C. (2012). Propuesta para promover el espiritu emprendedor a través de la educación. Perú: Centro de Competitividad y Estudios Estratégicos de IPAE Acción Empresarial.

Filion, L. (2000). El oficio del emprendedor: pensar y actuar como un emprendedor. Caracas: Infoem, AJE.

Hernández, et al. (2010). Metodologia de la investigacion. México. D.F: McGraw Hill.

Hernández, T. y Lacuesta, G. (s. f.). Aplicación del aprendizaje basado en problemas (PBL) bajo un enfoque multidisciplinar: una experiencia práctica. Recuperado el 23 de noviembre de 2014 http://dialnet.unirioja.es/ servlet/articulo?codigo $=2232506$

Jaramillo, L. (2008). Emprendimiento: concepto básico en competencias. Lumen: Universidad del Norte, Instituto de Estudios en Educación. 
Ministerio de Educación de Chile (2009). Cuaderno de gestión Imagina, atrévete a emprender. Chile: Pontificia Universidad Católica de Chile y Fundación Chile, Asistencia Técnica de la Unión Europea.

Orti, A. M.y Clares, J. (2007). Metodologías formativas en los sistemas de capacitación de emprendedores. Sevilla: Universidad de Sevilla, Dialnet.

Spencer, L. M.y Spencer, S. M. (1993). Competence at work. Models of superior performance. New York: John Wiley \& Sons.

Tamayo, M. (2011). El proceso de la investigación cientifica. México: Limusa S.A.

Tobón, S. (2006). Formación basada en competencias. Pensamiento complejo, diseño curricular y didáctica. Bogotá: ECOE Ediciones.

Vainrub, R. (2007). Convertir sueños en realidades. Una guía para emprendedores. Caracas: Ediciones IESA.

Zabalza, M. (2003). Competencias docentes del profesorado universitario. Madrid: Nancea, S.A. de Ediciones. 